Cosmological electromagnetic fields and dark energy

This article has been downloaded from IOPscience. Please scroll down to see the full text article.

JCAP03(2009)016

(http://iopscience.iop.org/1475-7516/2009/03/016)

View the table of contents for this issue, or go to the journal homepage for more

Download details:

IP Address: 147.96.14.15

The article was downloaded on 01/07/2013 at 15:50

Please note that terms and conditions apply. 


\title{
Cosmological electromagnetic fields and dark energy
}

\author{
Jose Beltrán Jiménez and Antonio L. Maroto \\ Departamento de Física Teórica I, Universidad Complutense de Madrid, \\ 28040 Madrid, Spain \\ E-mail: jobeltra@fis.ucm.es, maroto@fis.ucm.es
}

Received December 3, 2008

Revised February 11, 2009

Accepted February 17, 2009

Published March 10, 2009

\begin{abstract}
We show that the presence of a temporal electromagnetic field on cosmological scales generates an effective cosmological constant which can account for the accelerated expansion of the universe. Primordial electromagnetic quantum fluctuations produced during electroweak scale inflation could naturally explain the presence of this field and also the measured value of the dark energy density. The behavior of the electromagnetic field on cosmological scales is found to differ from the well studied short-distance behavior and, in fact, the presence of a non-vanishing cosmological constant could be signalling the breakdown of gauge invariance on cosmological scales. The theory is compatible with all the local gravity tests, and is free from classical or quantum instabilities. Thus we see that, not only the true nature of dark energy can be established without resorting to new physics, but also the value of the cosmological constant finds a natural explanation in the context of standard inflationary cosmology. This mechanism could be discriminated from a true cosmological constant by upcoming observations of CMB anisotropies and large scale structure.
\end{abstract}

Keywords: dark energy theory, quantum field theory on curved space 


\section{Contents}

1 Introduction 1

2 Cosmological electromagnetic fields $\quad 2$

3 Quantum fluctuations during inflation 4

$\begin{array}{lll}4 & \text { Gauge invariance on cosmological scales } & 7\end{array}$

5 Perturbations and consistency $\quad 8$

6 Conclusions and discussion $\quad 10$

\section{Introduction}

The nature of dark energy, which is believed to be responsible for the present phase of accelerated expansion of the universe [1-4], still remains unknown. Despite its phenomenological success, the simplest description in terms of a cosmological constant $(\Lambda \mathrm{CDM}$ model) suffers from an important naturalness problem, since the measured value of $\Lambda$, corresponding to $\rho_{\Lambda} \sim \rho_{M} \sim\left(2 \times 10^{-3} \mathrm{eV}\right)^{4}$, finds no natural explanation in the context of known physics. Moreover, the fact that today matter and dark energy have comparable contributions to the energy density, turns out to be difficult to understand if dark energy is a true cosmological constant. Thus, the energy density of a cosmological constant remains constant throughout the history of the universe, whereas those of the rest of components (matter or radiation) grow as we go back in time. Then the question arises as to whether it is a coincidence (or not) that they have comparable values today when they have differed by many orders of magnitude in the past. Notice also that if $\Lambda$ is a fundamental constant of nature, its scale (around $10^{-3} \mathrm{eV}$ ) is more than 30 orders of magnitude smaller than the natural scale of gravitation, $G=M_{P}^{-2}$ with $M_{P} \sim 10^{19} \mathrm{GeV}$. On the other hand, if $\Lambda$ is just an effective parametrization of dark energy, still a proper understanding of the underlying physics would be needed in order to explain the measured value.

Alternative models have been proposed in which dark energy is a dynamical component rather than a cosmological constant. Such models are usually based on new physics, either in the form of new cosmological fields or modifications of Einstein's gravity [5-9]. However, they are generically plagued by classical or quantum instabilities, fine tuning problems or inconsistencies with local gravity constraints.

In this paper we explore the possibility of understanding dark energy from the standard electromagnetic field, without the need of introducing new physics (previous works on models of dark energy based on vector fields can be found in [10-17]). We will show that the behavior of electromagnetic fields on very large (super-Hubble) scales differs from the well studied short-distance (sub-Hubble) behavior. Thus, on super-Hubble scales, the time component of the electromagnetic field grows linearly in time in the matter and radiation eras, giving rise to a cosmological constant contribution in the electromagnetic energy-momentum tensor 
(the potential gravitational effects of longitudinal electromagnetic fields were considered in a different context in [18]). At late times this contribution becomes dominant giving rise to the accelerated phase. As a possible generating mechanism, we calculate the spectrum of super-Hubble electromagnetic modes produced during inflation from quantum fluctuations and find that the correct value of the dark energy density can be naturally obtained in the case in which inflation took place at the electroweak scale.

\section{Cosmological electromagnetic fields}

We start by writing the electromagnetic action including a gauge-fixing term in the presence of gravity:

$$
S=\int d^{4} x \sqrt{-g}\left[-\frac{1}{16 \pi G} R-\frac{1}{4} F_{\mu \nu} F^{\mu \nu}+\frac{\lambda}{2}\left(\nabla_{\mu} A^{\mu}\right)^{2}\right]
$$

The gauge-fixing term is required in order to define a consistent quantum theory for the electromagnetic field [19], and we will see that it plays a fundamental role on large scales. Still this action preserves a residual gauge symmetry $A_{\mu} \rightarrow A_{\mu}+\partial_{\mu} \phi$ with $\square \phi=0$.

Einstein's and electromagnetic equations derived from this action can be written as:

$$
\begin{aligned}
R_{\mu \nu}-\frac{1}{2} R g_{\mu \nu} & =8 \pi G\left(T_{\mu \nu}+T_{\mu \nu}^{A}\right) \\
\nabla_{\nu} F^{\mu \nu}+\lambda \nabla^{\mu} \nabla_{\nu} A^{\nu} & =0
\end{aligned}
$$

where $T_{\mu \nu}$ is the energy-momentum tensor for matter and radiation and $T_{\mu \nu}^{A}$ is the energymomentum tensor of the electromagnetic field. Notice that since we will be using the covariant Gupta-Bleuler formalism, we do not a priori impose the Lorenz condition. The effect of the high conductivity of the universe in the matter and radiation eras will be discussed below.

We shall first focus on the simplest case of a homogeneous electromagnetic field (zero mode) in a flat Robertson-Walker background, whose metric is given by:

$$
d s^{2}=d t^{2}-a(t)^{2} \delta_{i j} d x^{i} d x^{j}
$$

In this space-time, equations (2.3) read:

$$
\begin{aligned}
\ddot{A}_{0}+3 H \dot{A}_{0}+3 \dot{H} A_{0} & =0 \\
\ddot{\vec{A}}+H \dot{\vec{A}} & =0
\end{aligned}
$$

with $H=\dot{a} / a$ the Hubble parameter.

Notice that (2.5) implies that the gauge-fixing term exactly behaves as a cosmological constant throughout the history of the universe, irrespective of the background evolution. Indeed, for homogeneous fields we have:

$$
\frac{d}{d t}\left(\nabla_{\mu} A^{\mu}\right)=\frac{d}{d t}\left(\dot{A}_{0}+3 H A_{0}\right)=0
$$

We can solve (2.5) and (2.6) during the radiation and matter dominated epochs when the Hubble parameter is given by $H=p / t$ with $p=1 / 2$ for radiation and $p=2 / 3$ for matter. In such a case the solutions for (2.6) are:

$$
\begin{aligned}
A_{0}(t) & =A_{0}^{+} t+A_{0}^{-} t^{-3 p} \\
\vec{A}(t) & =\vec{A}^{+} t^{1-p}+\vec{A}^{-}
\end{aligned}
$$


where $A_{0}^{ \pm}$and $\vec{A}^{ \pm}$are constants of integration. Hence, the growing mode of the temporal component does not depend on the epoch being always proportional to the cosmic time $t$, whereas the growing mode of the spatial component evolves as $t^{1 / 2}$ during radiation and as $t^{1 / 3}$ during matter, i.e. at late times the temporal component will dominate over the spatial ones.

On the other hand, the $\left(\begin{array}{ll}{ }^{3} & 0\end{array}\right)$ component of Einstein's equations adopts the following form:

$$
H^{2}=\frac{8 \pi G}{3}\left[\sum_{\alpha=R, M} \rho_{\alpha}+\rho_{A_{0}}+\rho_{\vec{A}}\right]
$$

where $R, M$ stands for radiation and matter respectively and:

$$
\begin{aligned}
\rho_{A_{0}} & =\lambda\left(\frac{9}{2} H^{2} A_{0}^{2}+3 H A_{0} \dot{A}_{0}+\frac{1}{2} \dot{A}_{0}^{2}\right) \\
\rho_{\vec{A}} & =\frac{1}{2 a^{2}}(\dot{\vec{A}})^{2}
\end{aligned}
$$

Notice that we need $\lambda>0$ in order to have positive energy density for $A_{0}$. Besides, when inserting the solutions (2.8) and (2.9) into these expressions we obtain that $\rho_{A_{0}}=\rho_{A_{0}}^{0}$, $\rho_{\vec{A}}=\rho_{\vec{A}}^{0} a^{-4}$ and $\nabla_{\mu} A^{\mu}=$ const as commented before. Thus, the field behaves as a cosmological constant throughout the evolution of the universe since its temporal component gives rise to a constant energy density whereas the energy density corresponding to $\vec{A}$ always decays as radiation. Moreover, this fact prevents the generation of a non-negligible anisotropy which could spoil the highly isotropic CMB radiation (see [20] for a more general discussion). Finally, when the universe is dominated by the cosmological constant arising from the gaugefixing term, both the Hubble parameter and $A_{0}$ become constant leading therefore to a future de Sitter universe. Let us emphasize that according to (2.7), $\rho_{A_{0}}$ always contributes as a cosmological constant. As the observed fraction of energy density associated to a cosmological constant today is $\Omega_{\Lambda} \simeq 0.7$, we obtain that the field value today must be $A_{0}\left(t_{0}\right) \simeq 0.3 M_{P}$.

The effects of the high electric conductivity $\sigma$ can be introduced using the magnetohydrodynamical approximation and including on the r.h.s. of Maxwell's equations the corresponding current term, which is given by $J_{\mu}-J_{\nu} u^{\nu} u_{\mu}=\sigma F_{\mu \nu} u^{\nu}$ with $u^{\mu}$ the velocity associated to the comoving observers. Notice that the strict neutrality of the plasma, which is consistent with a vanishing electric field, implies $J_{\mu} u^{\mu}=0$, and finally, the current can be written as: $J_{\mu}=\left(0, \sigma\left(\partial_{0} A_{i}-\partial_{i} A_{0}\right)\right)$. Notice that electric neutrality also implies that conductivity does not affect the evolution of $A_{0}(t)$. The infinite conductivity limit simply eliminates the growing mode of $\vec{A}(t)$ in (2.9). The inhomogeneous case, corresponding to $k \neq 0$ modes, will be discussed in next section.

We still need to understand which are the appropriate initial conditions leading to the present value of $A_{0}$. In order to avoid the cosmic coincidence problem, such initial conditions should have been set in a natural way in the early universe. In a very interesting work [21], it was suggested that the present value of the dark energy density could be related to physics at the electroweak scale since $\rho_{\Lambda} \sim\left(M_{\mathrm{EW}}^{2} / M_{P}\right)^{4}$, where $M_{\mathrm{EW}} \sim 10^{3} \mathrm{GeV}$. This relation offers a hint on the possible mechanism generating the initial amplitude of the electromagnetic fluctuations. Indeed, we see that if such amplitude is set by the size of the Hubble horizon at the electroweak era, i.e. $A_{0}\left(t_{\mathrm{EW}}\right)^{2} \sim H_{\mathrm{EW}}^{2}$, then the correct scale for the dark energy density is obtained. Thus, using the Friedmann equation, we find $H_{\mathrm{EW}}^{2} \sim M_{\mathrm{EW}}^{4} / M_{P}^{2}$, but according to $(2.11), \rho_{A_{0}} \sim H^{2} A_{0}^{2} \sim$ const., so that $\rho_{A_{0}} \sim H_{\mathrm{EW}}^{4} \sim\left(M_{\mathrm{EW}}^{2} / M_{P}\right)^{4}$ as commented before. 
A possible implementation of this mechanism can take place during inflation. Notice that the typical scale of the dispersion of quantum field fluctuations on super-Hubble scales generated in an inflationary period is precisely set by the almost constant Hubble parameter during such period $H_{I}$, i.e. $\left\langle A_{0}^{2}\right\rangle \sim H_{I}^{2}$ [22]. The correct dark energy density can then be naturally obtained if initial conditions for the electromagnetic fluctuations are set during an inflationary epoch at the scale $M_{I} \sim M_{\mathrm{EW}}$. Let us make these arguments more precise.

\section{Quantum fluctuations during inflation}

We shall look at the electromagnetic perturbations generated during inflation in order to determine its primordial power spectrum. In this case it is more convenient to use conformal time $\eta$ defined by means of $d t=a d \eta$ and to introduce the conformal components of the field $\mathcal{A}_{\mu}=\left(a A_{0}, \vec{A}\right)$. Besides we shall focus on a single Fourier mode of the vector field with wave vector $\vec{k}$ and decompose the field in temporal, transverse and longitudinal components with respect to $\vec{k}$. In this frame, equations (2.3) read:

$$
\begin{aligned}
\mathcal{A}_{0 k}^{\prime \prime}-\left[\frac{k^{2}}{\lambda}-2 \mathcal{H}^{\prime}+4 \mathcal{H}^{2}\right] \mathcal{A}_{0 k}-2 i k\left[\frac{1+\lambda}{2 \lambda} \mathcal{A}_{\| k}^{\prime}-\mathcal{H} \mathcal{A}_{\| k}\right] & =0 \\
\overrightarrow{\mathcal{A}}_{\perp k}^{\prime \prime}+k^{2} \overrightarrow{\mathcal{A}}_{\perp k} & =a \sigma \overrightarrow{\mathcal{A}}_{\perp k}^{\prime} \\
\mathcal{A}_{\| k}^{\prime \prime}-k^{2} \lambda \mathcal{A}_{\| k}-2 i k \lambda\left[\frac{1+\lambda}{2 \lambda} \mathcal{A}_{0 k}^{\prime}+\mathcal{H} \mathcal{A}_{0 k}\right] & =a \sigma\left(\mathcal{A}_{\| k}^{\prime}-i k \mathcal{A}_{0 k}\right)
\end{aligned}
$$

with ' $\equiv \frac{d}{d \eta}$ and $\mathcal{H}=a H$ is the Hubble parameter in conformal time. He have included for completeness the current term on the right-hand side as commented before. Notice that once again the electric neutrality of the universe implies that the evolution equation for the temporal component is not modified. During inflation the electric conductivity of the universe is negligible and this term can be safely neglected so that in the following we shall set $\sigma=0$.

It is easy to see from equations (3.1) that the transverse modes are just plane waves irrespective of the expansion rate. On the other hand, the components $\mathcal{A}_{0 k}$ and $\mathcal{A}_{\| k}$ are coupled to each other even in the absence of gravity. This is due to the fact that we are working with arbitrary $\lambda$ and not using the simple Feynman gauge $\lambda=-1$.

Let us first consider quantization in Minkowski space-time, with $\mathcal{H}=\mathcal{H}^{\prime}=0$. The decomposition in Fourier modes can be written as follows:

$$
\begin{aligned}
\mathcal{A}_{0}=\int \frac{d^{3} \vec{k}}{2 k_{0}(2 \pi)^{3}}[ & \left(-i \frac{1+\lambda}{1-\lambda}\left(\mathbf{a}_{0}+\mathbf{a}_{\|}\right) k \eta+\mathbf{a}_{0}\right) e^{-i k x} \\
& \left.+\left(i \frac{1+\lambda}{1-\lambda}\left(\mathbf{a}_{0}^{+}+\mathbf{a}_{\|}^{+}\right) k \eta+\mathbf{a}_{0}^{+}\right) e^{i k x}\right] \\
\mathcal{A}_{\|}=\int \frac{d^{3} \vec{k}}{2 k_{0}(2 \pi)^{3}}[ & \left(i \frac{1+\lambda}{1-\lambda}\left(\mathbf{a}_{0}+\mathbf{a}_{\|}\right) k \eta+\mathbf{a}_{\|}\right) e^{-i k x} \\
& \left.+\left(-i \frac{1+\lambda}{1-\lambda}\left(\mathbf{a}_{0}^{+}+\mathbf{a}_{\|}^{+}\right) k \eta+\mathbf{a}_{\|}^{+}\right) e^{i k x}\right]
\end{aligned}
$$

with $k_{0}=|\vec{k}|=k$. Now, in order to have the canonical commutation rules $\left[\mathcal{A}_{\mu}(t, \vec{x}), \Pi^{\nu}(t, \vec{y})\right]=i \delta_{\mu}^{\nu} \delta^{(3)}(\vec{x}-\vec{y})$, the creation and annihilation operators appearing in (3.2) 
should satisfy:

$$
\begin{aligned}
& {\left[\mathbf{a}_{0}(\vec{k}), \mathbf{a}_{0}^{+}\left(\overrightarrow{k^{\prime}}\right)\right]=\frac{1-\lambda}{\lambda} k_{0}(2 \pi)^{3} \delta^{(3)}\left(\vec{k}-\overrightarrow{k^{\prime}}\right)} \\
& {\left[\mathbf{a}_{\|}(\vec{k}), \mathbf{a}_{\|}^{+}\left(\overrightarrow{k^{\prime}}\right)\right]=-\frac{1-\lambda}{\lambda} k_{0}(2 \pi)^{3} \delta^{(3)}\left(\vec{k}-\overrightarrow{k^{\prime}}\right)}
\end{aligned}
$$

For simplicity in the following we will take $\lambda=1 / 3$ so that we use canonically normalized operators with positive sign for the temporal component. Notice that this is just the opposite situation to the usual Feynman gauge. In fact, $\lambda=1 / 3$ and $\lambda=-1$ are the only two possible choices with canonical normalizations. As is well-known [19], in order to recover Maxwell's theory, we need to eliminate the negative norm states by defining the corresponding restricted Hilbert space. Following the standard Gupta-Bleuler formalism, the physical states $|\phi\rangle$ will be those annihilated by the combination $\mathbf{a}_{0}+\mathbf{a}_{\|}$, that is: $\left(\mathbf{a}_{0}(\vec{k})+\mathbf{a}_{\|}(\vec{k})\right)|\phi\rangle=0$. In Minkowski space-time, only transverse degrees of freedom contribute to the expectation value of the energy density in the physical states and $\left\langle\phi\left|T_{00}\right| \phi\right\rangle>0$ since the contributions from longitudinal and temporal modes cancel each other. Thus, as expected, the theory is free from ghosts. Notice that in Minkowski space-time, we also get $\left\langle\phi\left|\partial_{\mu} A^{\mu}\right| \phi\right\rangle=0$.

Now we can proceed to the quantization in the inflationary epoch. In order to present the calculational method explicitly, we assume an exact de Sitter phase. The general quaside Sitter results will be given below. Thus in de-Sitter: $a=-1 /(H \eta)$ and $\mathcal{H}=-1 / \eta$ with $\eta<0$. The classical solutions of the corresponding equations are a bit more complicated, although it is still possible to obtain analytic expressions:

$$
\begin{aligned}
& \mathcal{A}_{0 k}=C_{1} k \eta e^{-i k \eta}+\frac{C_{2}}{k \eta}\left[\frac{1}{2}(1+i k \eta) e^{-i k \eta}-k^{2} \eta^{2} e^{i k \eta} E_{1}(2 i k \eta)\right] \\
& \mathcal{A}_{\| k}=i C_{1}(1+i k \eta) e^{-i k \eta}-i C_{2}\left[\frac{3}{2} e^{-i k \eta}+(1-i k \eta) e^{i k \eta} E_{1}(2 i k \eta)\right]
\end{aligned}
$$

where $E_{1}(x)=\int_{1}^{\infty} e^{-t x} / t d t$ is the exponential integral function. Note that the mode $C_{1}$ can be gauged away by means of a residual gauge transformation. The sub-Hubble limit $(|k \eta| \gg 1)$ of these solutions reads:

$$
\begin{aligned}
& \mathcal{A}_{0 k}=\left(C_{1} k \eta+i C_{2}\right) e^{-i k \eta} \\
& \mathcal{A}_{\| k}=\left(-C_{1} k \eta-i C_{2}\right) e^{-i k \eta}
\end{aligned}
$$

The choice of adiabatic vacuum [23] is made by matching these solutions with those obtained in the Minkowski case (3.2) (up to sub-leading terms in the sub-Hubble limit). To do this, we choose the modes $C_{1}$ and $C_{2}$ in the following way:

$$
\begin{aligned}
C_{1} & \rightarrow-\frac{i}{k_{0}}\left(\mathbf{a}_{0}+\mathbf{a}_{\|}\right) \\
C_{2} & \rightarrow-\frac{i}{2 k_{0}} \mathbf{a}_{0}
\end{aligned}
$$

On the other hand, on super-Hubble scales $(|k \eta| \ll 1)$ we have:

$$
\begin{aligned}
& \mathcal{A}_{0 k}=\frac{1}{2} C_{2}(k \eta)^{-1} \\
& \mathcal{A}_{\| k}=i C_{1}-i C_{2}\left(\frac{3}{2}-\gamma-\ln (2 i k \eta)\right)
\end{aligned}
$$


with $\gamma$ the Euler's constant. We see that $\mathcal{A}_{0 k} \propto a$, which means that $A_{0 k}=a^{-1} \mathcal{A}_{0 k}$ is (almost) constant during inflation, once the mode leaves the horizon. In the case of quasi-de Sitter slow-roll inflation, the Hubble parameter reads $\mathcal{H}=-1 /((1-\varepsilon) \eta)$, where the slow-roll parameter is defined as $\varepsilon=1 /(16 \pi G)\left(V^{\prime} / V\right)^{2} \ll 1$, with $V$ the inflaton potential. Following the same steps as before, we obtain the power spectrum for $A_{0}$ on super-Hubble scales:

$$
\mathcal{P}_{A_{0}}(k) \equiv \frac{k^{3}}{2 \pi^{2}}\left\langle\left|A_{0 k}\right|^{2}\right\rangle=\frac{H_{I}^{2}}{16 \pi^{2}}\left[\frac{k}{a H_{I}}\right]^{n_{A_{0}}}
$$

which is almost scale-invariant (as in the scalar field case) since for the electromagnetic spectral index we obtain $n_{A_{0}}=-4 \varepsilon$. In a similar way it is possible to obtain the primordial power spectrum of longitudinal modes on super-Hubble scales:

$$
\mathcal{P}_{A_{\|}}(k)=\frac{k^{2}}{16 \pi^{2} \varepsilon^{2}}\left[\frac{k}{a H_{I}}\right]^{-4 \varepsilon}
$$

If we now compare the power spectra for the conformal fields $\mathcal{A}_{0}$ and $\mathcal{A}_{\|}$we find that:

$$
\frac{\mathcal{P}_{\mathcal{A}_{\|}}(k)}{\mathcal{P}_{\mathcal{A}_{0}}(k)}=\frac{1}{\varepsilon^{2}}\left(\frac{k}{a H_{I}}\right)^{2}
$$

which is negligible on super-Hubble scales, and allows us to safely ignore the longitudinal modes on such scales after inflation.

Notice that since $\varepsilon>0, \mathcal{P}_{A_{0}}(k)$ is a red-tilted spectrum which means that the contribution to $\left\langle A_{0}^{2}\right\rangle$ from long wavelenghts dominates over small scales. In particular, provided inflation lasted for a sufficiently large number of e-folds, this allows to decompose the fluctuations field at any given time into a large homogeneous contribution (with scales $k<\mathcal{H}$ ) and a small inhomogeneous perturbation $(k>\mathcal{H})$, and therefore we can use standard perturbation theory around the homogeneous background. Thus, for the homogeneous part we get:

$$
\left\langle A_{0}^{2}\right\rangle_{\text {hom }}=\int_{k_{\min }}^{k_{*}} \frac{d k}{k} \mathcal{P}_{A_{0}}(k) \simeq H_{I}^{2} \frac{e^{-n_{A_{0}} \tilde{N}}}{16 \pi^{2}\left|n_{A_{0}}\right|}
$$

where $k_{*} \lesssim H_{0}, \tilde{N}=N_{\text {tot }}-N_{0}$ and $k_{\min }=e^{-\tilde{N}} H_{0}$ is set by the Hubble horizon at the beginning of inflation [24]. Here $N_{\text {tot }}$ is the total number of e-folds of inflation which should not be confused with $N_{0}$ which is the number of e-folds since the time when the scale $H_{0}^{-1}$ left the horizon. Typical values for $N_{0}$ are around 50, whereas generically there is no upper limit to $N_{\text {tot }}$. Thus as expected, up to tilt corrections, $H_{I}$ sets the scale for the field dispersion.

Once the fluctuations generated during inflation enter the radiation dominated era, it would be in principle possible that the high conductivity of the universe could modify their evolution, spoiling the growing behavior of super-Hubble models found in (2.8). However since conductivity does not affect the temporal equation, the $A_{0}$ modes are not modified on super-Hubble scales. In figure 1 we show the evolution of super-Hubble temporal and longitudinal modes, both for vanishing and infinite conductivity. We see that the evolution exactly corresponds to $\mathcal{A}_{0 k} \propto \eta^{3}$ as expected from (2.8) in the radiation era, even in the infinite conductivity case. We see that $\mathcal{A}_{\| k}$ is sub-dominant compared to $\mathcal{A}_{0 k}$, until the modes re-enter the Hubble radius for $|k \eta| \simeq 1$. The result is not sensitive to the change of initial conditions. Thus, the only effect of the high conductivity is the damping of the electric field,(which is consistent with the strict neutrality of the plasma). In particular, 


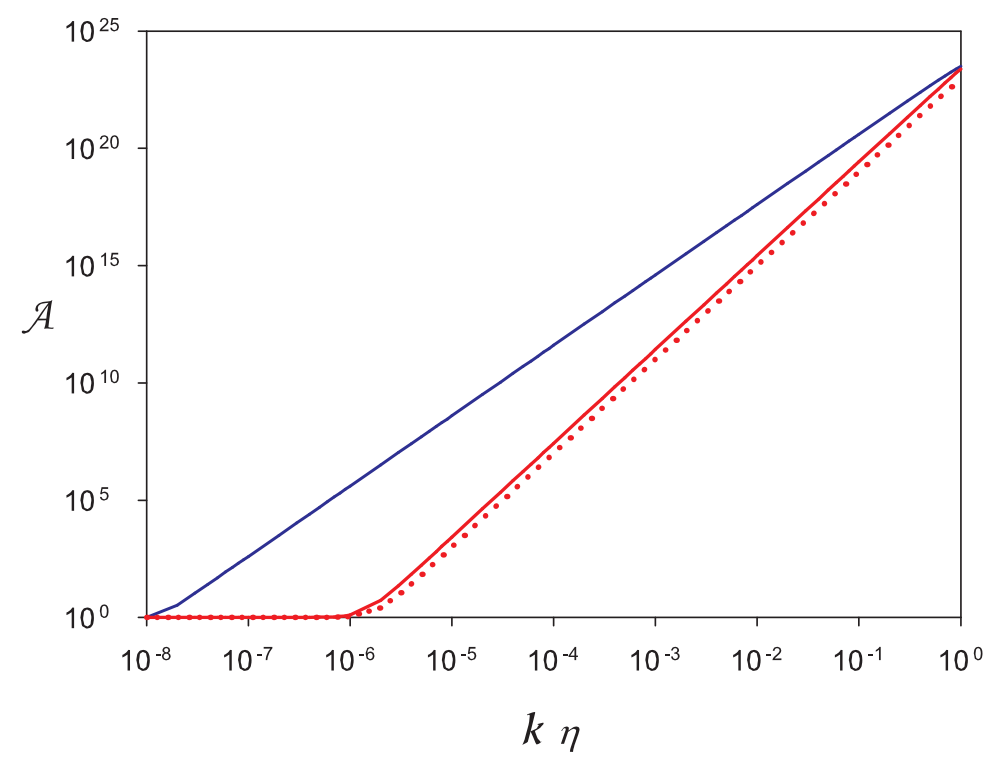

Figure 1. Evolution of $\mathcal{A}_{0 k}$ and $\mathcal{A}_{\| k}$ on super-Hubble scales in the radiation era. Continuous (dotted) blue lines correspond to $\mathcal{A}_{0 k}$ for infinite (vanishing) conductivity (no difference in the plot). Continuous (dotted) red lines correspond to $\mathcal{A}_{\| k}$ for infinite (vanishing) conductivity.

this implies $\mathcal{A}_{\| k}^{\prime}=i k \mathcal{A}_{0 k}$, which corresponds to the field evolution shown in figure 1 . Let us emphasize that the vanishing of the electric field does not imply the vanishing of the temporal component $\mathcal{A}_{0 k}$.

In fact, it is straightforward to show that the value of $\nabla_{\mu} A^{\mu}$ giving rise to the effective cosmological constant is not affected by the presence of conductivity. Indeed, Maxwell's equations in the presence of conserved currents read:

$$
\nabla_{\nu} F^{\mu \nu}+\lambda \nabla^{\mu} \nabla_{\nu} A^{\nu}=J^{\mu}
$$

Taking the four-divergence of the equation we get:

$$
\square\left(\nabla_{\mu} A^{\mu}\right)=0
$$

where we have used current conservation $\nabla_{\mu} J^{\mu}=0$. Thus, we see that the field $\nabla_{\mu} A^{\mu}$ evolves as a free scalar field, and it is therefore constant on super-Hubble scales, independently of the presence of external currents.

\section{Gauge invariance on cosmological scales}

A remarkable consequence of the covariant quantization formalism in the context of inflationary cosmology (which had not been considered previously) is the breaking of gauge invariance on cosmological scales. Indeed, in this formalism, the classical energy-momentum tensor depends on the gauge-fixing term. However, in Minkowski space-time, when one takes the expectation value of this object in a physical state (that belonging to the restricted Hilbert 
space) the gauge dependence disappears because the contributions from the temporal and longitudinal degrees of freedom cancel each other. This is so just because in Minkowski space-time, in the restricted Hilbert space, the amplitudes of temporal and longitudinal mode solutions are exactly the same (see [19]). However when considering the quantization in an expanding universe important differences arise. At short distances, i.e. for sub-Hubble modes, it is easy to see from (3.5) that the same cancellation takes place, as it should be, and the theory is exactly the same as in Minkowski space-time. Therefore the energy density does not depend on the gauge-fixing term. Nevertheless, when the modes become super-Hubble, it can be seen from (3.7), that the amplitude of the temporal modes grows in time faster than that of the longitudinal ones. This spoils the mentioned cancellation and a net energy density results from the $\lambda$ term.

Notice that in the covariant formalism the four polarizations are always present. In Minkowski space-time (or for sub-Hubble modes) only two of them contribute to the energy density, however on cosmological scales also the temporal one can have observational consequences. As $A_{0}$ is also a propagating degree of freedom, the gauge-fixing term can be seen in the Gupta-Bleuler formalism as a kinetic term for it, and therefore the coefficient $\lambda$ can be fixed by the standard normalization of the creation and annihilation operators. This effect could not be studied in other (non-covariant) formalisms, such as Coulomb gauge quantization, since only transverse polarizations would be present in that case.

To summarize, the presence of the background cosmological electromagnetic field breaks $\mathrm{U}(1)_{\mathrm{EM}}$ symmetry on large scales while preserving local (small-scales) invariance. This is analogous to the situation with Lorentz symmetry, where the presence of matter or radiation in the Universe breaks global Lorentz invariance, but respecting local transformations. In other words, the presence of a non-vanishing cosmological constant could be signalling the breakdown of gauge invariance on cosmological scales. Let us emphasize that this effect is a consequence of the quantization of electromagnetic theory in the covariant formalism and, as discussed above, it does not modify any of the physical predictions of Maxwell's theory for laboratory experiments or astrophysical observations. As a matter of fact, the electromagnetic interaction has not been tested on distance scales larger than 1.3 AU [25].

\section{$5 \quad$ Perturbations and consistency}

Despite the fact that the background evolution in the present case is the same as in $\Lambda \mathrm{CDM}$, the evolution of metric perturbations could be different, thus offering an observational way of discriminating between the two models. With this purpose, we have calculated the evolution of metric, matter density and electromagnetic perturbations in the longitudinal gauge with $\delta g_{00}=2 a^{2} \Phi, \delta g_{0 i}=a^{2} S_{i}, \delta g_{i j}=a^{2}\left(2 \Psi \delta_{i j}-h_{i j}\right), \delta=\delta \rho_{M} / \rho_{M}$ and taking $\mathcal{A}_{\mu}=\mathcal{A}_{\mu}^{\text {hom }}(\eta)+\delta \mathcal{A}_{\mu}$, where as commented before the main contribution to $\mathcal{A}_{\mu}^{\text {hom }}(\eta)$ comes from the temporal component. The propagation speeds of scalar, vector and tensor perturbations are found to be real and equal to the speed of light, so that the theory is classically stable. We have also checked that the theory does not contain ghosts and it is therefore stable at the quantum level. On the other hand, using the explicit expressions in [26] for the vector-tensor theory of gravity corresponding to the action in (2.1), it is possible to see that all the parametrized post-Newtonian (PPN) parameters agree with those of General Relativity, i.e. the theory is compatible with all the local gravity constraints for any value of the homogeneous background electromagnetic field. 


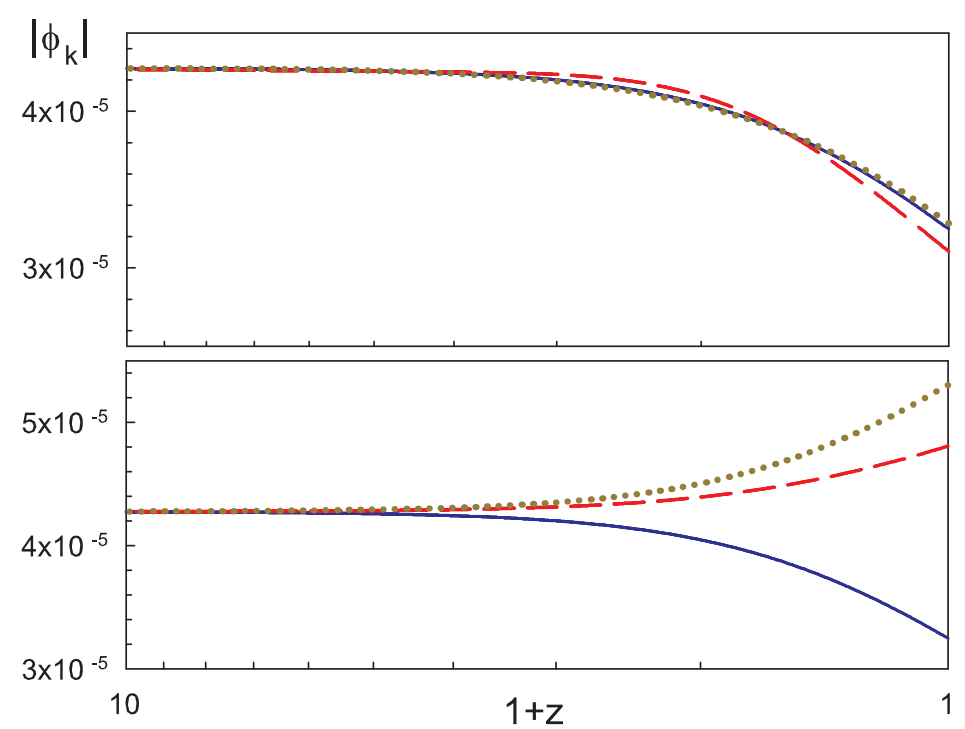

Figure 2. Evolution of $\Phi_{k}$ with $k=3 H_{0}$, corresponding to the maximum contribution to the ISW effect. Upper(lower) panel with vanishing(infinite) conductivity. Continuous blue line for $\Lambda$ CDM, dashed red for $\tilde{N}\left|n_{A_{0}}\right|=12$ (upper panel) 18 (lower panel) and dotted green for large $\tilde{N}\left|n_{A_{0}}\right|$.

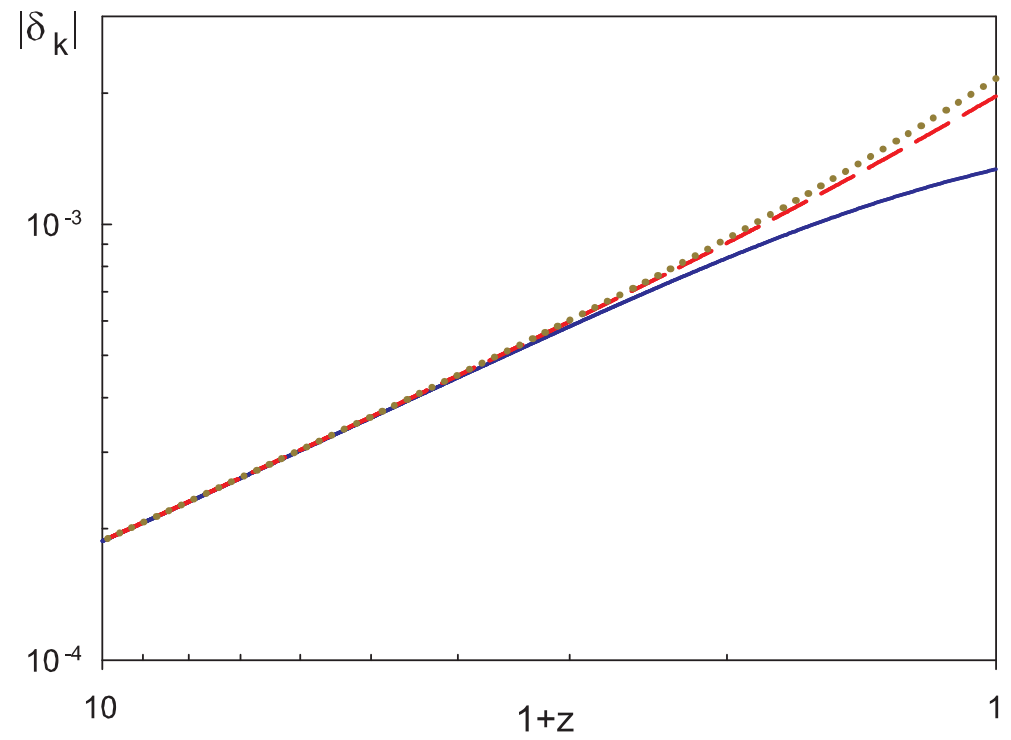

Figure 3. Evolution of the matter density contrast $\delta_{k}$ with $k=10 H_{0}$ and infinite conductivity (vanishing conductivity shows no difference with respect to $\Lambda \mathrm{CDM}$ ). Plotted curves correspond to the same models as in the lower panel of figure2.

In figures 2-3 we plot the evolution of scalar perturbations, satisfying $\Phi_{k}=\Psi_{k}$ in this theory, and matter density contrast $\delta_{k}$, in both, vanishing and infinite conductivity limits. We find that the only relevant deviations with respect to $\Lambda \mathrm{CDM}$ appear on large scales $k \sim H_{0}$ and that they depend on the primordial spectrum of electromagnetic fluctuations. However, for $\tilde{N}\left|n_{A_{0}}\right| \gtrsim 12$, the results on the CMB temperature power spectrum and evolution of den- 
sity perturbation are compatible with observations. Taking $M_{I} \sim T_{\mathrm{RH}} \sim 10^{2} \mathrm{GeV}$ in this case, with $T_{\mathrm{RH}}$ the reheating temperature, we obtain the correct value of the dark energy density today. In addition, the different evolution of $\Phi_{k}$ with respect to the $\Lambda$ CDM model gives rise to a possible discriminating contribution to the late-time integrated Sachs-Wolfe effect [27].

The presence of large scale electric fields generated by inhomogeneities in the $A_{0}$ field opens also the possibility for the generation of large scale currents which in turn could contribute to the presence of magnetic fields with large coherence scales. This could shed light on the problem of explaining the origin of cosmological magnetic fields. Work is in progress in this direction.

\section{Conclusions and discussion}

We have shown that the present phase of accelerated expansion of the universe can be explained by the presence of a cosmological electromagnetic field generated during inflation. This result not only offers a solution to the problem of establishing the true nature of dark energy, but also explains the value of the cosmological constant without resorting to new physics. In this scenario the fact that matter and dark energy densities coincide today is just a consequence of inflation taking place at the electroweak scale. Such a relatively low inflation scale implies also that no cosmological gravity wave background is expected to be measurable in future CMB polarization observations.

Notice also that any vector-tensor theory (not necessarily electromagnetism) whose low-energy effective action is given by (2.1) and in which the vector field only interacts gravitationally with the rest of particles would provide a natural model for dark energy. In fact all the previous models trying to account for the cosmic acceleration are plagued by classical or quantum instabilities, fine tuning problems or inconsistencies with Solar System experiments. However, in this work we present, for the first time, an explanation to the cosmic acceleration with none of the aforementioned problems.

\section{Acknowledgments}

We would like to thank J.D. Barrow, J.A.R. Cembranos and C. Tamarit for useful comments and suggestions. This work has been supported by DGICYT (Spain) project numbers FPA 2004-02602 and FPA 2005-02327, UCM-Santander PR34/07-15875, CAM/UCM 910309 and MEC grant BES-2006-12059.

\section{References}

[1] Supernova Cosmology Project collaboration, S. Perlmutter et al., Measurements of $\Omega$ and $\Lambda$ from 42 high-redshift supernovae, Astrophys. J. 517 (1999) 565 [astro-ph/9812133] [SPIRES].

[2] Supernova Search Team collaboration, A.G. Riess et al., Observational evidence from supernovae for an accelerating universe and a cosmological constant, Astron. J. 116 (1998) 1009 [astro-ph/9805201] [SPIRES]; BV RI light curves for 22 type Ia supernovae, Astron. J. 117 (1999) 707 [astro-ph/9810291] [SPIRES].

[3] WMAP collaboration, D.N. Spergel et al. First-year Wilkinson Microwave Anisotropy Probe (WMAP) observations: determination of cosmological parameters, Astrophys. J. Suppl. 148 (2003) 175 [astro-ph/0302209] [SPIRES]; 
WMAP collaboration, D.N. Spergel et al., Wilkinson Microwave Anisotropy Probe (WMAP) three year results: implications for cosmology, Astrophys. J. Suppl. 170 (2007) 377 [astro-ph/0603449] [SPIRES].

[4] SDSS collaboration, M. Tegmark et al., Cosmological parameters from SDSS and WMAP, Phys. Rev. D 69 (2004) 103501 [astro-ph/0310723] [SPIRES].

[5] C. Wetterich, Cosmology and the fate of dilatation symmetry, Nucl. Phys. B 302 (1988) 668 [SPIRES].

[6] R.R. Caldwell, R. Dave and P.J. Steinhardt, Cosmological imprint of an energy component with general equation-of-state, Phys. Rev. Lett. 80 (1998) 1582 [astro-ph/9708069] [SPIRES].

[7] C. Armendariz-Picon, T. Damour and V.F. Mukhanov, k-Inflation, Phys. Lett. B 458 (1999) 209 [hep-th/9904075] [SPIRES].

[8] S.M. Carroll, V. Duvvuri, M. Trodden and M.S. Turner, Is cosmic speed-up due to new gravitational physics?, Phys. Rev. D 70 (2004) 043528 [astro-ph/0306438] [SPIRES].

[9] G.R. Dvali, G. Gabadadze and M. Porrati, 4D gravity on a brane in $5 D$ Minkowski space, Phys. Lett. B 485 (2000) 208 [hep-th/0005016] [SPIRES].

[10] V.V. Kiselev, Vector field as a quintessence partner, Class. Quant. Grav. 21 (2004) 3323 [gr-qc/0402095] [SPIRES].

[11] C. Armendariz-Picon, Could dark energy be vector-like?, JCAP 07 (2004) 007 [astro-ph/0405267] [SPIRES].

[12] C.G. Boehmer and T. Harko, Dark energy as a massive vector field, Eur. Phys. J. C 50 (2007) 423 [gr-qc/0701029] [SPIRES].

[13] M. Novello, S.E. Perez Bergliaffa and J. Salim, Nonlinear electrodynamics and the acceleration of the universe, Phys. Rev. D 69 (2004) 127301 [astro-ph/0312093] [SPIRES].

[14] T.S. Koivisto and D.F. Mota, Vector field models of inflation and dark energy, JCAP 08 (2008) 021 [arXiv:0805.4229] [SPIRES].

[15] H.S. Zhao, Coincidences of dark energy with dark matter: clues for a simple alternative?, Astrophys. J. 671 (2007) L1 [arXiv:0710.3616] [SPIRES].

[16] J.B. Jiménez and A.L. Maroto, A cosmic vector for dark energy, Phys. Rev. D 78 (2008) 063005 [arXiv:0801.1486] [SPIRES]; Vector models for dark energy, arXiv:0807.2528 [SPIRES].

[17] K. Bamba, S. Nojiri and S.D. Odintsov, Inflationary cosmology and the late-time accelerated expansion of the universe in non-minimal Yang-Mills- $F(R)$ gravity and non-minimal vector-F $(R)$ gravity, Phys. Rev. D 77 (2008) 123532 [arXiv:0803.3384] [SPIRES].

[18] S. Deser, The limit of massive electrodynamics, Ann. Inst. Henri Poincaré 16 (1972) 79 [SPIRES].

[19] C. Itzykson and J.B. Zuber, Quantum field theory, McGraw-Hill, U.S.A. (1980).

[20] J.D. Barrow, Cosmological limits on slightly skew stresses, Phys. Rev. D 55 (1997) 7451 [gr-qc/9701038] [SPIRES];

J.D. Barrow and R. Maartens, Anisotropic stresses in inhomogeneous universes, Phys. Rev. D 59 (1999) 043502 [astro-ph/9808268] [SPIRES].

[21] N. Arkani-Hamed, L.J. Hall, C.F. Kolda and H. Murayama, A new perspective on cosmic coincidence problems, Phys. Rev. Lett. 85 (2000) 4434 [astro-ph/0005111] [SPIRES].

[22] A. Linde, Particle physics and inflationary cosmology, Harwood Academic Press (1996).

[23] N.D. Birrell and P.C.W. Davies, Quantum fields in curved space, Cambridge U.K. (1982). 
[24] A.R. Liddle and D.H. Lyth, Cosmological inflation and large-scale structure, Cambridge U.K. (2000).

[25] A.S. Goldhaber and M.M. Nieto, Photon and graviton mass limits, arXiv:0809.1003 [SPIRES].

[26] C. Will, Theory and experiment in gravitational physics, Cambridge University Press, Cambridge U.K. (1993).

[27] R.G. Crittenden and N. Turok, Looking for $\Lambda$ with the Rees-Sciama effect, Phys. Rev. Lett. 76 (1996) 575 [astro-ph/9510072] [SPIRES]. 